\title{
ON THE NUMERICAL SOLUTION OF FRACTIONAL PARTIAL DIFFERENTIAL EQUATIONS
}

\author{
Solat Karimi Vanani and Azim Aminataei \\ Department of Mathematics, K. N. Toosi University of Technology, P.O. Box: 16315- \\ 1618, Tehran, Iran \\ solatkarimi@yahoo.com, ataei@kntu.ac.ir
}

\begin{abstract}
In this paper, a technique generally known as meshless method is presented for solving fractional partial differential equations (FPDEs). Some physical linear and nonlinear experiments such as time-fractional convective-diffusion equation, timefractional wave equation and nonlinear space-fractional Fisher's equation are considered. We present the advantages of using the radial basis functions (RBFs) especially wherein the data points are scattered. Comparing between the numerical results obtained from our method and the other methods confirms the good accuracy of the presented scheme.
\end{abstract}

Key Words- Radial basis functions, Fractional partial differential equations

\section{INTRODUCTION}

The theory of fractional calculus was first raised in the year 1695 by Marquis de L'Hopital and from now on many studies were done and many important books were published in this field wherein we can point out to the books of Oldham and Spanier [1], Miller and Ross [2], Samko et al. [3] and Podlubny [4]. Most of the scientific problems and phenomena are modeled by FPDEs. For instance, in mathematical physics [4] in fluid and continuum mechanics [5], colored noises [6], biology, chemistry, acoustics and psychology [7]. Some of FPDEs including the fractional Fokker-Planck equation [8], the fractional KdV equation [9] and linear and nonlinear space- and time-fractional diffusion-wave equation $[10,11]$ have been studied and solved. In most cases, these problems do not admit analytical solution, so these equations should be solved using special techniques. In the last decade, several computational methods have been applied to solve FDEs, prominent among which are the homotopy perturbation method (HPM) $[12,13]$, the Adomian decomposition method (ADM) [14, 15], the variational iteration method (VIM) [12], the generalized differential transformation method (GDTM) [16] and the fractional difference method (FDM) $[17,18]$.

In the present research work, we are desired to present a truly meshless approximation strategy for solving FPDEs based on RBFs.

In the last two decades, the use of RBFs for both interpolation and for solving PDEs have received considerable attention in various fields of research and attracted many researchers to solve the problems in higher-dimensional spaces. Because the RBFs as a class of mesh-free schemes avoid grid generation and the domain of interest can be considered by a set of scattered data points among which there is no pre-defined connectivity. This method of solution is effective on scattered data points and in irregular geometries, is easy to implement in any finite dimension, and is spectrally 
accurate. The application of RBFs to solve PDEs was first used in [19, 20] and then was applied to solve various problems arising in many sciences especially in physics and mechanics. For instance; PDEs using in viscoelastic flows [21], PDEs using in natural frequencies of composite plates [22], Klein-Gordon equation using in solid state physics, plasma physics, fluid dynamics [23], KdV equation using in pressure, waves, liquid-gas bubble mixture, and wave phenomena inharmonic crystals [24], delay differential systems arising in various areas [25] and etc.

The present paper has been organized as follows: Section 2 gives notations and basic definitions of the fractional calculus. In Section 3, we introduce some basic aspects of RBF interpolation and its development to solve FPDEs. Some experiments and their results are presented and compared with several other methods in Section 4. Finally in Section 5, the study is concluded and the findings are summarized.

\section{BASIC DEFINITIONS OF THE FRACTIONAL CALCULUS}

In this Section, we state some preliminaries and definitions of fractional calculus [4]. Definition 1. A real function $u(x), x>0$ is said to be in the space $C_{\mu}, \mu \in R$, if there exists a real number $\mathrm{p}>\mu$ such that $\mathrm{u}(\mathrm{x})=\mathrm{x}^{\mathrm{p}} \mathrm{v}(\mathrm{x})$, where $\mathrm{v}(\mathrm{x}) \in \mathrm{C}[0, \infty)$ and it is said to be in the space $C_{\mu}^{m}$ iff $u^{(m)}(x) \in C_{\mu}, m \in N$.

Definition 2. The Riemann-Liouville fractional integral operator of order $\alpha \geq 0$, of a function $\mathrm{u}(\mathrm{x}) \in \mathrm{C}_{\mu}, \mu \geq-1$, is defined as:

$\mathrm{J}^{\alpha} \mathrm{u}(\mathrm{x})=\frac{1}{\Gamma(\alpha)} \int_{0}^{\mathrm{x}}(\mathrm{x}-\mathrm{t})^{\alpha-1} \mathrm{u}(\mathrm{t}) \mathrm{dt}, \quad \alpha>0, \mathrm{x}>0$,

$\mathrm{J}^{0} \mathrm{u}(\mathrm{x})=\mathrm{u}(\mathrm{x})$,

where $\Gamma$ is the Gamma function.

Definition 3. The fractional derivative of $\mathrm{u}(\mathrm{x})$ in the Caputo's sense is defined as:

$D^{\alpha} u(x)=J^{m-\alpha} D^{m} u(x)=\frac{1}{\Gamma(m-\alpha)} \int_{0}^{x}(x-t)^{m-\alpha-1} u^{(m)}(t) d t$,

where $\mathrm{m}-1<\alpha \leq \mathrm{m}, \mathrm{m} \in \mathrm{N}, \mathrm{x}>0, \mathrm{u}(\mathrm{x}) \in \mathrm{C}_{-1}^{\mathrm{m}}$.

Definition 4. For $m$ to be the smallest integer that exceeds $\alpha$, the Caputo's spacefractional derivative operator of order $\alpha>0$ is defined as:

$\mathrm{D}_{\mathrm{x}}^{\alpha} \mathrm{u}(\mathrm{x}, \mathrm{t})= \begin{cases}\frac{1}{\Gamma(\mathrm{m}-\alpha)} \int_{0}^{\mathrm{x}}(\mathrm{x}-\sigma)^{\mathrm{m}-\alpha-1} \frac{\partial^{\mathrm{m}} \mathrm{u}(\sigma, \mathrm{t})}{\partial \sigma^{\mathrm{m}}} \mathrm{d} \sigma, & \mathrm{m}-1<\alpha<\mathrm{m}, \\ \frac{\partial^{\mathrm{m}} \mathrm{u}(\mathrm{x}, \mathrm{t})}{\partial \mathrm{x}^{\mathrm{m}}}, & \alpha=\mathrm{m},\end{cases}$

and the time-fractional derivative operator of order $\alpha>0$ is defined as:

$$
\mathrm{D}_{\mathrm{t}}^{\alpha} \mathrm{u}(\mathrm{x}, \mathrm{t})= \begin{cases}\frac{1}{\Gamma(\mathrm{m}-\alpha)} \int_{0}^{\mathrm{t}}(\mathrm{t}-\tau)^{\mathrm{m}-\alpha-1} \frac{\partial^{\mathrm{m}} \mathrm{u}(\mathrm{x}, \tau)}{\partial \tau^{\mathrm{m}}} \mathrm{d} \tau, & \mathrm{m}-1<\alpha<\mathrm{m}, \\ \frac{\partial^{\mathrm{m}} \mathrm{u}(\mathrm{x}, \mathrm{t})}{\partial \mathrm{t}^{\mathrm{m}}}, & \alpha=\mathrm{m} .\end{cases}
$$




\section{RADIAL BASIS FUNCTIONS AND THEIR APPLICATIONS ON FRACTIONAL PARTIAL DIFFERENTIAL EQUATIONS}

To explain the concepts of RBFs, we need assert some preliminaries and some notations from [26].

Let the function $\mathrm{u}(\mathbf{x})$ is defined as $\mathrm{u}(\mathbf{x}): \mathrm{R}^{\mathrm{d}} \rightarrow \mathrm{R}$ and suppose that the approximation $\hat{\mathrm{u}}(\mathbf{x})$ at an arbitrary point $\mathbf{x}$ can be written as a linear combination of $\mathrm{n}$ basis functions in the following form:

$$
\mathrm{u}(\mathbf{x}) \cong \hat{\mathrm{u}}(\mathbf{x})=\sum_{\mathrm{i}=1}^{\mathrm{n}} \mathrm{u}_{\mathrm{i}} \phi_{\mathrm{i}}(\mathbf{x}), \quad \mathbf{x} \in \Omega \subset \mathrm{R}^{\mathrm{d}},
$$

where the set of RBFs $\left\{\phi_{\mathrm{i}}(\mathbf{x})\right\}_{\mathrm{i}=1}^{\mathrm{n}}=\left\{\phi\left(\left\|\mathbf{x}-\mathbf{x}_{\mathrm{i}}\right\|, \mathrm{c}_{\mathrm{i}}\right)\right\}_{\mathrm{i}=1}^{\mathrm{n}}$ is chosen in advance and the set of $\left\{u_{i}\right\}_{i=1}^{n}$ is to be find. Also, $n$ is the number of nodal points fallen within the influence domain $\Omega$ of $\mathbf{x}$ and $\mathrm{c}_{\mathrm{i}}$ denotes the shape parameter. The most important and useful RBFs are as follows:

$$
\begin{array}{ll}
\text { i. Euclidean: } & \phi_{\mathrm{i}}(\mathbf{x})=\left\|\mathbf{x}-\mathbf{x}_{\mathrm{i}}\right\|, \\
\text { ii. Multiquadric: } & \phi_{\mathrm{i}}(\mathbf{x})=\sqrt{\left\|\mathbf{x}-\mathbf{x}_{\mathrm{i}}\right\|^{2}+\mathrm{c}_{\mathrm{i}}^{2}},
\end{array}
$$

iii. Inverse multiquadric: $\phi_{i}(\mathbf{x})=\frac{1}{\sqrt{\left\|\mathbf{x}-\mathbf{x}_{i}\right\|^{2}+c_{i}^{2}}}$,

iv. Thin-plate spline: $\quad \phi_{\mathrm{i}}(\mathbf{x})=\left\|\mathbf{x}-\mathbf{x}_{\mathrm{i}}\right\|^{2} \log \left\|\mathbf{x}-\mathbf{x}_{\mathrm{i}}\right\|$,

$$
\text { v. Gauss kernel: } \quad \phi_{\mathrm{i}}(\mathbf{x})=\mathrm{e}^{-\left\|\mathbf{x}-\mathbf{x}_{\mathrm{i}}\right\|^{2} / \mathrm{c}_{\mathrm{i}}^{2}} \text {. }
$$

The $\left\|\mathbf{x}-\mathbf{x}_{i}\right\|$ denotes the distance between $\mathbf{x}$ and the $\mathrm{i}$-th nodal point $\mathbf{x}_{\mathrm{i}}$ and the shape parameter can be defined as $c_{i}=\beta d_{i}$, where $\beta>0$ is a factor and $d_{i}$ is the distance from the $\mathrm{i}$-th collocation point to the nearest neighbouring collocation point $[27,28]$.

In this paper, we use two important RBFs, the multiquadric RBF (MQ-RBF) and inverse multiquadric RBF (IMQ-RBF) as the base functions to approximate the desired problems. The MQ-RBF and IMQ-RBF as infinitely smooth functions, are among the most popular RBFs. The MQ-RBF was first introduced by Hardy [29] who successfully applied it for approximating surface and bodies from field data. Hardy [30] has written a detailed review article summarizing its explosive growth in use since it was first introduced. In 1972, Franke [31] published a detailed comparison of 29 different scattered data schemes against analytic problems. Of all the techniques tested, he concluded that MQ-RBF performed the best in accuracy, visual appeal, and ease of implementation, even against various finite element schemes. The MQ-RBF and IMQRBF approximation schemes are grid-free spatial approximation schemes which converge exponentially for the spatial terms of ODEs and PDEs and offer many computational advantages over traditional methods. Some of the most advantages of 
MQ-RBF and IMQ-RBF schemes are that they are truly mesh-free schemes which possess very high order rates of convergence [32].

Now, the main goal is to evaluate the coefficients $\left\{\mathrm{u}_{\mathrm{i}}\right\}_{\mathrm{i}=1}^{\mathrm{n}}$ in equation (4). Therefore, we substitute the collocation points $\left\{\mathbf{x}_{\mathrm{j}}\right\}_{\mathrm{j}=1}^{\mathrm{n}}$ within the domain $\Omega$ in equation (4). So, for $\mathrm{j}=1,2, \ldots, \mathrm{n}$; we obtain:

$\hat{\mathrm{u}}\left(\mathbf{x}_{\mathrm{j}}\right)=\sum_{\mathrm{i}=1}^{\mathrm{n}} \mathrm{u}_{\mathrm{i}} \phi_{\mathrm{i}}\left(\mathbf{x}_{\mathrm{j}}\right)=\mathrm{u}_{1} \phi_{1}\left(\mathbf{x}_{\mathrm{j}}\right)+\mathrm{u}_{2} \phi_{2}\left(\mathbf{x}_{\mathrm{j}}\right)+\ldots+\mathrm{u}_{\mathrm{n}} \phi_{\mathrm{n}}\left(\mathbf{x}_{\mathrm{j}}\right)$.

The operational matrix form of equation (5) can be written in the following manner: $\hat{\mathrm{u}}=\underline{\Phi} \underline{\mathrm{u}}$,

where

$\hat{\mathrm{u}}=\left[\hat{\mathrm{u}}\left(\mathbf{x}_{1}\right), \hat{\mathrm{u}}\left(\mathbf{x}_{2}\right), \ldots, \hat{\mathrm{u}}\left(\mathbf{x}_{\mathrm{n}}\right)\right]^{\mathrm{T}}, \quad \underline{\mathrm{u}}=\left[\mathrm{u}_{1}, \mathrm{u}_{2}, \ldots, \mathrm{u}_{\mathrm{n}}\right]^{\mathrm{T}}$,

and $\underline{\Phi}$ is a coefficient matrix as:

$\Phi=\left(\begin{array}{cccccc}\phi_{1}\left(\mathbf{x}_{1}\right) & \phi_{2}\left(\mathbf{x}_{1}\right) & \cdots & \phi_{\mathrm{i}}\left(\mathbf{x}_{1}\right) & \cdots & \phi_{\mathrm{n}}\left(\mathbf{x}_{1}\right) \\ \phi_{1}\left(\mathbf{x}_{2}\right) & \phi_{2}\left(\mathbf{x}_{2}\right) & \cdots & \phi_{\mathrm{i}}\left(\mathbf{x}_{2}\right) & \cdots & \phi_{\mathrm{n}}\left(\mathbf{x}_{2}\right) \\ \vdots & \vdots & \ddots & \vdots & \ddots & \vdots \\ \phi_{1}\left(\mathbf{x}_{\mathrm{i}}\right) & \phi_{2}\left(\mathbf{x}_{\mathrm{i}}\right) & \cdots & \phi_{\mathrm{i}}\left(\mathbf{x}_{\mathrm{i}}\right) & \cdots & \phi_{\mathrm{i}}\left(\mathbf{x}_{\mathrm{i}}\right) \\ \vdots & \vdots & \ddots & \vdots & \ddots & \vdots \\ \phi_{1}\left(\mathbf{x}_{\mathrm{n}}\right) & \phi_{2}\left(\mathbf{x}_{\mathrm{n}}\right) & \cdots & \phi_{\mathrm{i}}\left(\mathbf{x}_{\mathrm{n}}\right) & \cdots & \phi_{\mathrm{n}}\left(\mathbf{x}_{\mathrm{n}}\right)\end{array}\right)$.

$\underline{\Phi}$ is an $\mathrm{n} \times \mathrm{n}$ real symmetric matrix.

Theoretical results show that the RBF interpolation matrix is nonsingular, whatever the number of the data sites and therefore $\underline{\Phi}^{-1}$ exists [33].

Hence, we can obtain $\underline{u}$ as:

$\underline{\mathrm{u}}=\underline{\Phi}^{-1} \hat{\mathrm{u}}$.

Let us cosider $\varphi$ as:

$\underline{\varphi}=\left[\phi_{1}(\mathbf{x}), \phi_{2}(\mathbf{x}), \ldots, \phi_{\mathrm{n}}(\mathbf{x})\right]$,

we can rewrite equation (4) as:

$\hat{\mathrm{u}}(\mathbf{x})=\underline{\varphi} \underline{\mathrm{u}}=\varphi \underline{\Phi}^{-1} \hat{\mathrm{u}}=\underline{\Psi} \hat{\mathrm{u}}$,

where

$\underline{\Psi}=\underline{\varphi} \underline{\Phi}^{-1}=\left[\psi_{1}, \psi_{2}, \ldots, \psi_{\mathrm{n}}\right]$.

The functions $\psi_{\mathrm{i}}, \mathrm{i}=1,2, \ldots, \mathrm{n}$; are the shape functions which satisfy the Kronecker delta condition:

$\psi_{\mathrm{i}}\left(\mathbf{x}_{\mathrm{j}}\right)=\left\{\begin{array}{ll}1, & \mathrm{i}=\mathrm{j}, \\ 0, & \mathrm{i} \neq \mathrm{j},\end{array} \quad \mathrm{i}, \mathrm{j}=1,2, \ldots, \mathrm{n}\right.$.

We can implement the RBF approximation scheme in two manners, direct RBF (DRBF) and indirect RBF (IRBF) [28]. In the DRBF, we first assume that the approximate solution is as equation (4) and then the derivative functions are calculated directly by differentiation of equation (4). In the IRBF for approximating function and its 
derivatives, the highest order derivative function $\mathrm{f}$ is first approximated in terms of RBFs. In this paper, we apply the DRBF.

Now, in order to apply the RBF approximation scheme for solving FPDEs, let us consider a FPDE in the form:

$\mathrm{D}^{\alpha} \mathrm{u}+\mathrm{Lu}=\mathrm{f}, \quad$ in $\Omega \subset \mathrm{R}^{\mathrm{d}}$,

$\mathrm{Bu}=\mathrm{g}, \quad$ on $\partial \Omega$,

where $d$ is the dimension, $\partial \Omega$ denotes the boundary of the domain $\Omega, L$ is the differential operator, $\mathrm{D}^{\alpha}$ is the fractional differential operator of order $\alpha$ that operates on the interior, and $B$ is an operator that specifies the boundary conditions. Both the $f$ and $\mathrm{g}: \mathrm{R}^{\mathrm{d}} \rightarrow \mathrm{R}$ are known functions.

Let $\left\{\mathbf{x}_{\mathrm{j}}\right\}_{\mathrm{j}=1}^{\mathrm{n}}$ be the $\mathrm{n}$ collocation points in $\Omega \cup \partial \Omega$. We assume the collocation points are arranged in such a way that the first $n_{1}$ points and the last $n_{B}$ points are in $\Omega$ and on $\partial \Omega$, respectively.

Substituting the collocation points into equations (7) and (8), we obtain:

$$
\begin{aligned}
& \sum_{\mathrm{i}=1}^{\mathrm{n}} \mathrm{u}_{\mathrm{i}}\left(\mathrm{D}^{\alpha}+\mathrm{L}\right) \phi_{\mathrm{i}}\left(\mathbf{x}_{\mathrm{j}}\right)=\mathrm{f}\left(\mathbf{x}_{\mathrm{j}}\right), \quad \mathrm{j}=1,2, \ldots, \mathrm{n}_{1}, \\
& \sum_{\mathrm{i}=1}^{\mathrm{n}} \mathrm{u}_{\mathrm{i}} \mathrm{B} \phi_{\mathrm{i}}\left(\mathbf{x}_{\mathrm{j}}\right)=\mathrm{g}\left(\mathbf{x}_{\mathrm{j}}\right), \quad \mathrm{j}=\mathrm{n}_{1}+1, \mathrm{n}_{1}+2, \ldots, \mathrm{n} .
\end{aligned}
$$

Therefore, we have the following system:

$(\underline{\Pi}, \underline{\mathrm{B}}) \underline{\mathrm{u}}=(\underline{\mathrm{f}}, \underline{\mathrm{g}})$,

where

$$
\begin{aligned}
& \underline{\Pi}=\left(\mathrm{D}^{\alpha}+\mathrm{L}\right) \underline{\Phi}=\left[\begin{array}{cccc}
\left(\mathrm{D}^{\alpha}+\mathrm{L}\right) \phi_{1}\left(\mathbf{x}_{1}\right) & \left(\mathrm{D}^{\alpha}+\mathrm{L}\right) \phi_{2}\left(\mathbf{x}_{1}\right) & \cdots & \left(\mathrm{D}^{\alpha}+\mathrm{L}\right) \phi_{\mathrm{n}}\left(\mathbf{x}_{1}\right) \\
\left(\mathrm{D}^{\alpha}+\mathrm{L}\right) \phi_{1}\left(\mathbf{x}_{2}\right) & \left(\mathrm{D}^{\alpha}+\mathrm{L}\right) \phi_{2}\left(\mathbf{x}_{2}\right) & \cdots & \left(\mathrm{D}^{\alpha}+\mathrm{L}\right) \phi_{\mathrm{n}}\left(\mathbf{x}_{2}\right) \\
\vdots & \vdots & \ddots & \vdots \\
\left(\mathrm{D}^{\alpha}+\mathrm{L}\right) \phi_{1}\left(\mathbf{x}_{\mathrm{n}_{1}}\right) & \left(\mathrm{D}^{\alpha}+\mathrm{L}\right) \phi_{2}\left(\mathbf{x}_{\mathrm{n}_{1}}\right) & \cdots & \left(\mathrm{D}^{\alpha}+\mathrm{L}\right) \phi_{\mathrm{n}}\left(\mathbf{x}_{\mathrm{n}_{1}}\right)
\end{array}\right] \text {, } \\
& \underline{\mathrm{B}}=\mathrm{B} \underline{\Phi}=\left[\begin{array}{cccc}
\mathrm{B} \phi_{1}\left(\mathbf{x}_{\mathrm{n}_{1}+1}\right) & \mathrm{B} \phi_{2}\left(\mathbf{x}_{\mathrm{n}_{1}+1}\right) & \cdots & \mathrm{B} \phi_{\mathrm{n}}\left(\mathbf{x}_{\mathrm{n}_{1}+1}\right) \\
\mathrm{B} \phi_{1}\left(\mathbf{x}_{\mathrm{n}_{1}+2}\right) & \mathrm{B} \phi_{2}\left(\mathbf{x}_{\mathrm{n}_{1}+2}\right) & \cdots & \mathrm{B} \phi_{\mathrm{n}}\left(\mathbf{x}_{\mathrm{n}_{1}+2}\right) \\
\vdots & \vdots & \ddots & \vdots \\
\mathrm{B} \phi_{1}\left(\mathbf{x}_{\mathrm{n}}\right) & \mathrm{B} \phi_{2}\left(\mathbf{x}_{\mathrm{n}}\right) & \cdots & \mathrm{B} \phi_{\mathrm{n}}\left(\mathbf{x}_{\mathrm{n}}\right)
\end{array}\right], \\
& \underline{\mathrm{f}}=\left[\mathrm{f}\left(\mathrm{x}_{1}\right), \mathrm{f}\left(\mathrm{x}_{2}\right), \ldots, \mathrm{f}\left(\mathrm{x}_{\mathrm{n}_{1}}\right)\right]^{\mathrm{T}} \text { and } \underline{\mathrm{g}}=\left[\mathrm{g}\left(\mathrm{x}_{\mathrm{n}_{1}+1}\right), \mathrm{g}\left(\mathrm{x}_{\mathrm{n}_{1}+2}\right), \ldots, \mathrm{g}\left(\mathrm{x}_{\mathrm{n}}\right)\right]^{\mathrm{T}} \text {. }
\end{aligned}
$$

Therefore, the system of $\mathrm{n}$ equations with $\mathrm{n}$ unknowns is available. Then, we must solve this system to make distinct the unknown coefficients. Hence, we have used the Gauss elimination method with total pivoting to solve such a system.

Remark. It is noticeable that collocating points can be scattered. This is one of the most important advantages of the RBF collocation method [20, 33]. In Section 4, the numerical results show this issue easily, and the applicability of the MQ-RBF and IMQ$\mathrm{RBF}$ approximation schemes in this sense, is observable. 


\section{ILLUSTRATIVE EXAMPLES}

In this Section, three examples including linear and nonlinear physical problems on regular and irregular domains are solved using MQ-RBF and IMQ-RBF. If the exact solution of the problem exists, the accuracy of an approximate solution is measured by means of the discrete relative $\mathrm{L}_{2}$ norm defined as:

$\mathrm{N}_{\mathrm{e}}=\left(\frac{\sum_{\mathrm{i}=1}^{\mathrm{n}}\left[\hat{\mathrm{u}}\left(\mathbf{x}_{\mathrm{i}}\right)-\mathrm{u}\left(\mathbf{x}_{\mathrm{i}}\right)\right]^{2}}{\sum_{\mathrm{i}=1}^{\mathrm{n}} \mathrm{u}^{2}\left(\mathbf{x}_{\mathrm{i}}\right)}\right)^{\frac{1}{2}}$,

where $\mathrm{u}$ and $\hat{\mathrm{u}}$ are the exact and computed solutions, respectively; and $\mathrm{n}$ is the number of unknown nodal values of $u$.

Since, most of FDEs have not exact solution, therefore we must compare them with known numerical methods such as HPM, VIM and GDTM . In all experiments, we assume that $\beta=10$. The results and their comparison with several powerful methods illustrate the validity and capability of these RBFs. The computations associated with the experiments were performed in Maple 13 on a PC, CPU $2.8 \mathrm{GHz}$.

\section{Example 4.1}

Consider the following linear time-fractional convective-diffusion equation [12]:

$\frac{\partial^{\alpha} \mathrm{u}}{\partial \mathrm{t}^{\alpha}}+\mathrm{x} \frac{\partial \mathrm{u}}{\partial \mathrm{x}}+\frac{\partial^{2} \mathrm{u}}{\partial \mathrm{x}^{2}}=2\left(1+\mathrm{t}+\mathrm{x}^{2}\right), \quad \mathrm{t}>0, \mathrm{x} \in \mathrm{R}, 0<\alpha \leq 1$,

with the initial condition: $\mathrm{u}(\mathrm{x}, 0)=\mathrm{x}^{2}$.

The exact solution is not known.

We have solved this problem by MQ-RBF with 25 points, $\alpha=0.75,0.85,0.95$ and have compared it with HPM to show the efficiency of the MQ-RBF. The results are given in Table 1.

Table 1: Comparison of the HPM and MQ-RBF approximation scheme for different $\mathrm{t}$ and $\mathrm{x}$ of the example 4.1

\begin{tabular}{|c|ccc|ccc|}
\hline \multicolumn{1}{|c|}{$t / X$} & \multicolumn{3}{|c|}{$\mathrm{u}_{\mathrm{HPM}}$} & \multicolumn{3}{c|}{$\mathrm{u}_{\text {MQ-RBF }}$} \\
\hline & $\alpha=0.75$ & $\alpha=0.85$ & $\alpha=0.95$ & $\alpha=0.75$ & $\alpha=0.85$ & $\alpha=0.95$ \\
\hline$t=0.1$ & & & & & & \\
0.00 & 0.025752 & 0.018043 & 0.019554 & 0.028367 & 0.018803 & 0.012705 \\
0.25 & 0.068252 & 0.070543 & 0.072054 & 0.090180 & 0.082325 & 0.075957 \\
0.50 & 0.255752 & 0.258043 & 0.259554 & 0.274098 & 0.269470 & 0.263539 \\
0.75 & 0.568252 & 0.570543 & 0.572054 & 0.587738 & 0.581273 & 0.574352 \\
1.00 & 1.005752 & 1.008043 & 1.009554 & 1.026875 & 1.019147 & 1.012597 \\
\hline$t=0.2$ & & & & & \\
0.00 & 0.061480 & 0.053140 & 0.044270 & 0.081070 & 0.061020 & 0.046272 \\
0.25 & 0.123980 & 0.115640 & 0.106770 & 0.142427 & 0.124613 & 0.109518 \\
0.50 & 0.311480 & 0.303140 & 0.294270 & 0.326059 & 0.311794 & 0.297085 \\
0.75 & 0.623980 & 0.615640 & 0.606770 & 0.639530 & 0.623601 & 0.607877 \\
1.00 & 1.061480 & 1.053140 & 1.044270 & 1.078591 & 1.061446 & 1.046098 \\
\hline
\end{tabular}


146 On The Numerical Solution of Fractional Partial Differential Equations

\begin{tabular}{|c|lll|lll|}
\hline \multicolumn{1}{|l|}{$t=0.3$} & & & & & \\
0.00 & 0.139965 & 0.119715 & 0.099492 & 0.157448 & 0.125825 & 0.100740 \\
0.25 & 0.202465 & 0.182215 & 0.161992 & 0.218462 & 0.189479 & 0.163979 \\
0.50 & 0.389965 & 0.369715 & 0.349492 & 0.401864 & 0.376697 & 0.351531 \\
0.75 & 0.702465 & 0.682215 & 0.661992 & 0.715194 & 0.688516 & 0.662301 \\
1.00 & 1.139965 & 1.119715 & 1.099492 & 1.154189 & 1.126349 & 1.100497 \\
\hline$t=0.4$ & & & & & & \\
0.00 & 0.240171 & 0.207205 & 0.175044 & 0.255900 & 0.212254 & 0.175812 \\
0.25 & 0.302671 & 0.269705 & 0.237544 & 0.316653 & 0.275962 & 0.239045 \\
0.50 & 0.490171 & 0.457205 & 0.425044 & 0.499869 & 0.463218 & 0.426583 \\
0.75 & 0.802671 & 0.769705 & 0.737544 & 0.813080 & 0.775056 & 0.737332 \\
1.00 & 1.240171 & 1.207205 & 1.175044 & 1.252017 & 1.212888 & 1.175502 \\
\hline$t=0.5$ & & & & & \\
0.00 & 0.361132 & 0.315087 & 0.270760 & 0.375034 & 0.319462 & 0.271226 \\
0.25 & 0.423632 & 0.377587 & 0.333260 & 0.435581 & 0.383221 & 0.334453 \\
0.50 & 0.611132 & 0.565087 & 0.520760 & 0.618646 & 0.570514 & 0.521977 \\
0.75 & 0.923632 & 0.877587 & 0.833260 & 0.931757 & 0.882374 & 0.832707 \\
1.00 & 1.361132 & 1.315087 & 1.270760 & 1.370641 & 1.320214 & 1.270852 \\
\hline
\end{tabular}

The computed results in Table 1, show a good agreement between the results obtained by MQ-RBF approximation scheme and HPM, again. This demonstrate the validity of MQ-RBF approximation scheme for FPDEs.

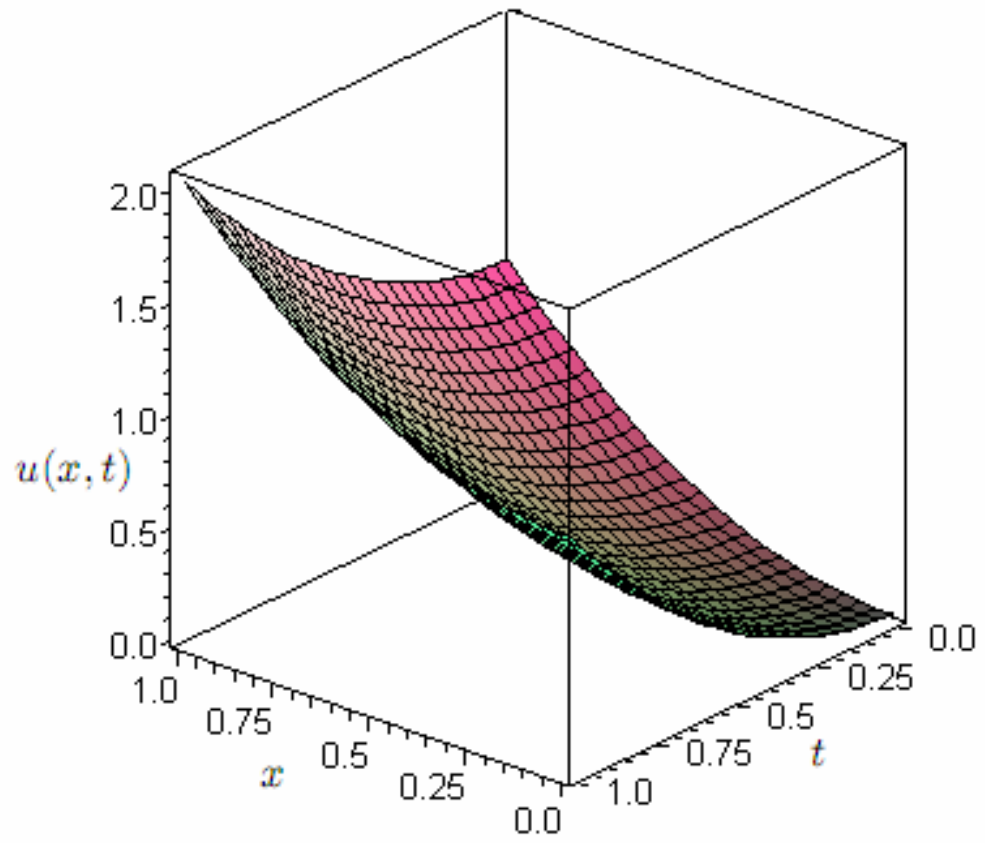

Figure 1. The graph of approximate solution of example 4.1 with $\alpha=0.75$ and $n=25$. 


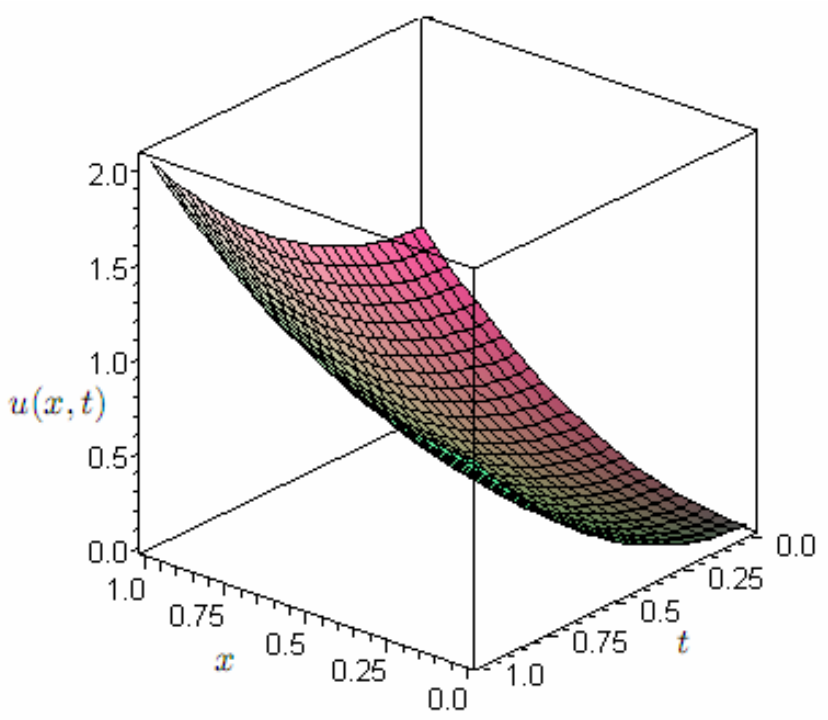

Figure 2. The graph of approximate solution of example 4.1 with $\alpha=0.85$ and $n=25$.

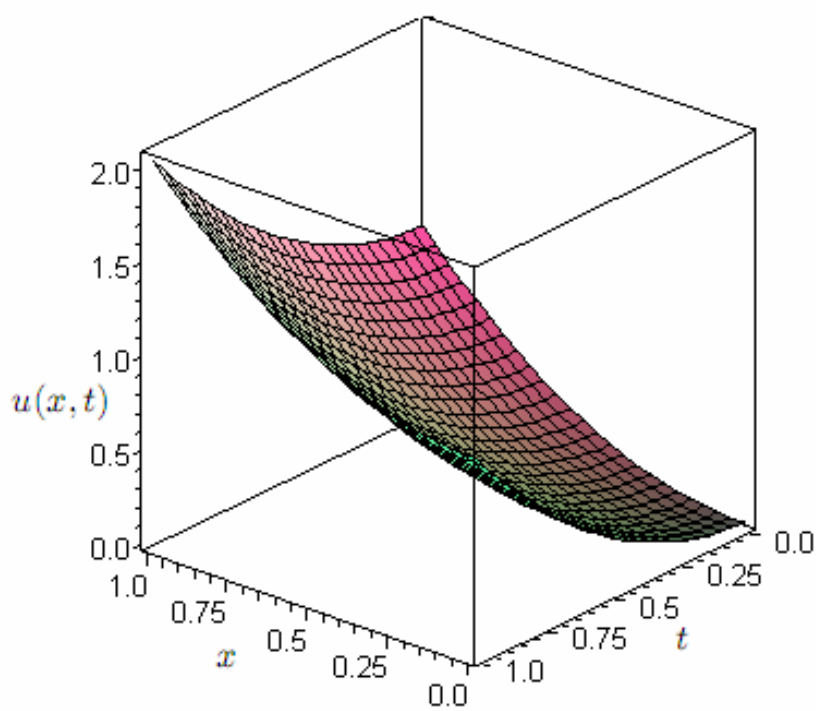

Figure 3. The graph of approximate solution of example 4.1 with $\alpha=0.95$ and $n=25$.

\section{Example 4.2}

Consider the following linear time-fractional wave equation [12, 34]:

$\frac{\partial^{\alpha} \mathrm{u}}{\partial \mathrm{t}^{\alpha}}=\frac{1}{2} \mathrm{x}^{2} \frac{\partial^{2} \mathrm{u}}{\partial \mathrm{x}^{2}}, \quad \mathrm{t}>0, \mathrm{x} \in \mathrm{R}, \quad 1<\alpha \leq 2$,

subject to initial conditions:

$\mathrm{u}(\mathrm{x}, 0)=\mathrm{x}, \quad$ and $\quad \frac{\partial \mathrm{u}(\mathrm{x}, 0)}{\partial \mathrm{t}}=\mathrm{x}^{2}$.

The exact solution is not known.

We have solved this problem by IMQ-RBF approximation scheme with 25 scattered data points, $\alpha=1.5$ and have compared it with VIM. 
Table 2: Comparison of the solutions of VIM and IMQ-RBF approximation scheme for scattered $\mathrm{x}$ and $\mathrm{t}$ of the example 4.2

\begin{tabular}{llllll}
\hline \multicolumn{5}{c}{$\mathbf{u}_{\text {VIM }}$} \\
\hline $\mathrm{x}$ & $\mathrm{t}=0$ & $\mathrm{t}=0.06$ & $\mathrm{t}=0.13$ & $\mathrm{t}=0.29$ & $\mathrm{t}=0.50$ \\
0.00 & 0.00 & 0.00 & 0.00 & 0.00 & 0.00 \\
0.11 & 0.11 & 0.110729 & 0.111595 & 0.113677 & 0.116726 \\
0.31 & 0.31 & 0.315791 & 0.322670 & 0.339207 & 0.363419 \\
0.88 & 0.88 & 0.926669 & 0.982101 & 1.115360 & 1.310469 \\
1.00 & 1.00 & 1.060265 & 1.131845 & 1.303926 & 1.555874 \\
\hline & & & & \\
\hline $\mathrm{x}$ & $\mathrm{t}=0$ & $\mathrm{t}=0.06$ & $\mathrm{t}=0.13$ & $\mathrm{t}$ (MB-RF & \\
0.00 & 0.00 & 0.00 & 0.00 & 0.00 & 0.00 \\
0.11 & 0.11 & 0.110729 & 0.111595 & 0.113679 & 0.116710 \\
0.31 & 0.31 & 0.315794 & 0.322675 & 0.339223 & 0.363298 \\
0.88 & 0.88 & 0.926696 & 0.982140 & 1.115490 & 1.309495 \\
1.00 & 1.00 & 1.060300 & 1.131896 & 1.304094 & 1.554617 \\
\hline
\end{tabular}

Here, the agreement of all data of $\mathrm{x}$ and $\mathrm{t}$ is observable for scattered data points. This is the biggest advantage of RBFs.

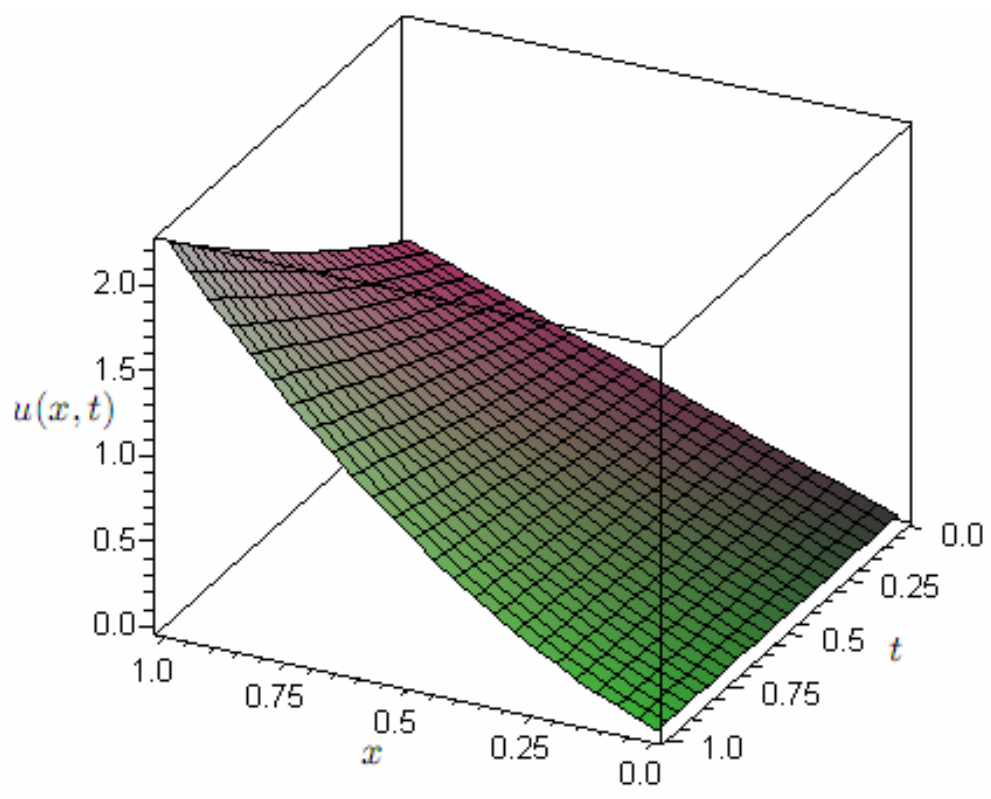

Figure 4. The graph of approximate solution of example 4.2 with $\alpha=1.5$ and $n=25$.

Example 4.3

Consider the following nonlinear space-fractional Fisher's equation [16, 35 ]:

$\frac{\partial \mathrm{u}}{\partial \mathrm{t}}-\frac{\partial^{1.5} \mathrm{u}}{\partial \mathrm{x}^{1.5}}-\mathrm{u}(\mathrm{x}, \mathrm{t})(1-\mathrm{u}(\mathrm{x}, \mathrm{t}))=\mathrm{x}^{2}, \quad \mathrm{x}>0$,

with the initial condition: $\mathrm{u}(\mathrm{x}, 0)=\mathrm{x}$.

The exact solution is not known.

We have solved this experiment by IMQ-RBF approximation scheme and have obtained the approximate solution with $n=49$. Comparison of the IMQ-RBF approximation scheme with GDTM [16] and VIM [35] for some points are given in Table 3. 
Table 3: Comparison of the solutions of GDTM, VIM and IMQ-RBF approximation scheme for different $\mathrm{t}$ and $\mathrm{x}$ of the example 4.3

\begin{tabular}{|c|c|c|c|c|c|c|}
\hline \multirow[t]{2}{*}{$X$} & \multicolumn{3}{|c|}{$t=0.1$} & \multicolumn{3}{|c|}{$t=0.2$} \\
\hline & $u_{G D T M}$ & $u_{V I M}$ & $U_{I M Q-R B F}$ & $u_{G D T M}$ & $u_{V I M}$ & $U_{I M Q-R B F}$ \\
\hline 0.0 & 0.000000 & 0.000000 & 0.000000 & 0.000000 & 0.000000 & 0.000000 \\
\hline 0.2 & 0.222033 & 0.220253 & 0.219761 & 0.240211 & 0.239550 & 0.240132 \\
\hline 0.4 & 0.439957 & 0.439860 & 0.438920 & 0.477796 & 0.477181 & 0.477550 \\
\hline 0.6 & 0.658707 & 0.658691 & 0.657719 & 0.711691 & 0.711815 & 0.712361 \\
\hline 0.8 & 0.876585 & 0.876757 & 0.875592 & 0.941795 & 0.943630 & 0.943940 \\
\hline 1.0 & 1.093587 & 1.094095 & 1.092409 & 1.168066 & 1.172881 & 1.171824 \\
\hline \multirow[t]{2}{*}{$x$} & \multicolumn{3}{|c|}{$t=0.3$} & \multicolumn{3}{|c|}{$t=0.4$} \\
\hline & $u_{G D T M}$ & $u_{V I M}$ & $u_{I M Q-R B F}$ & $u_{G D T M}$ & $u_{V I M}$ & $u_{I M Q-R B F}$ \\
\hline 0.0 & 0.000000 & 0.000000 & 0.000000 & 0.000000 & 0.000000 & 0.000000 \\
\hline 0.2 & 0.257431 & 0.255534 & 0.259207 & 0.269326 & 0.265626 & 0.275929 \\
\hline 0.4 & 0.509871 & 0.508336 & 0.512542 & 0.531749 & 0.529390 & 0.542191 \\
\hline 0.6 & 0.753428 & 0.754749 & 0.759926 & 0.777296 & 0.782568 & 0.798553 \\
\hline 0.8 & 0.987717 & 0.995443 & 1.000822 & 1.024978 & 1.026927 & 1.044459 \\
\hline 1.0 & 1.212587 & 1.231365 & 1.234069 & 1.214400 & 1.264908 & 1.277563 \\
\hline
\end{tabular}

From the above comparison, a good agreement between IMQ-RBF approximation scheme with the other methods is observable.

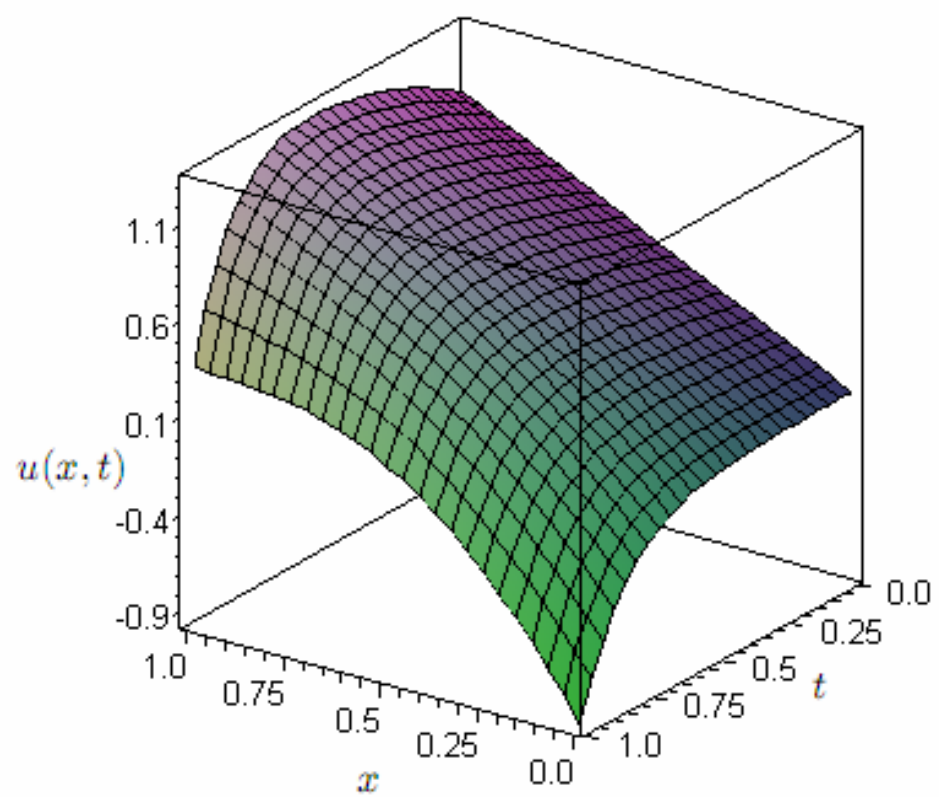

Figure 5. The graph of approximate solution of example 4.3 with $n=49$.

\section{CONCLUSION}

In this paper, the RBFs are applied for solving FPDEs using in engineering and physics. As the numerical examples show, the RBFs can deal with linear and nonlinear PDE problems with ease. For this, omel linear and nonlinear examples are numerically solved. Comparing between our results and other useful methods such as HPM, VIM and GDTM show the versatility, the capability and the efficiency of the RBFs. As we 
have observed, the method works excellently for scattered data points and irregular domains. Hence, these schemes are not depend on the selection of points. Also, this study has shown that the RBFs schemes can compete with the traditional methods as far as accuracy is concerned. It is hoped that the insights presented in this study will attract the attention of researchers to investigate the RBFs for the numerical solution of FPDEs.

\section{REFRENCES}

1. K.B. Oldham and J. Spanier, The fractional calculus, Academic Press, New York and London, 1974.

2. K.S. Miller and B. Ross, An introduction to the fractional calculus and fractional differential equations, John Wiley, New York, 1993.

3. S.G. Samko, A.A. Kilbas and O.I. Marichev, Fractional integrals and derivatives: theory and applications, Gordon and Breach Science Publishers, USA, 1993.

4. I. Podlubny, Fractional differential equations, Academic Press, New York, 1999.

5. F. Mainardi, Fractals and fractional calculus continuum mechanics, Springer Verlag, 291-348, 1997.

6. H.H. Sun, A.A. Abdelvahab and B. Onaral, Linear approximation of transfer function with a pole of fractional order, IEEE Trans. Automat. Control 29, 441-444, 1984.

7. W.M. Ahmad and R. El-Khazali, Fractional-order dynamical models of love. Chaos, Solitons \& Fractals 33, 1367-1375, 2007.

8. S. Chen, F. Liu, P. Zhuang and V. Anh, Finite difference approximations for the fractional Fokker-Planck equation, Applied Mathematical Modelling 33, 256-273, 2009.

9. S. Momani, An explicit and numerical solutions of the fractional $\mathrm{KdV}$ equation, Math. Comput. Simul. 70, 110-118, 2005.

10. S. Momani, Z. Odibat and V.S. Erturk, Generalized differential transform method for solving a space and time-fractional diffusion-wave equation, Physics Letters A 370, 379-387, 2007.

11. H. Jafari and S. Seifi, Homotopy analysis method for solving linear and nonlinear fractional diffusion-wave equation, Commun. Nonlinear Sci. Numer. Simulat. 14, 20062012, 2009.

12. S. Momani and Z. Odibat, Comparison between the homotopy perturbation method and the variational iteration method for linear fractional partial differential equations, Computers and Mathematics with Applications 54, 910-919, 2007.

13. H. Jafari and S. Seifi, Homotopy analysis method for solving linear and nonlinear fractional diffusion-wave equation, Commun. Nonlinear Sci. Numer. Simulat. 14, 20062012, 2009.

14. A.M.A. El-Sayed and M. Gaber, The Adomian decomposition method for solving partial differential equations of fractal order in finite domains, Physics Letters A 359, 175-182, 2006.

15. S. Momani and Z. Odibat, Analytical solution of a time-fractional Navier-Stokes equation by Adomian decomposition method, Appl. Math. Comput. 177, 488-494, 2006.

16. S. Momani and Z. Odibat, A novel method for nonlinear fractional partial differential equations: Combination of DTM and generalized Taylor's formula, Journal of Computational and Applied Mathematics 220, 85-95, 2008. 
17. S. Momani and Z. Odibat, Numerical comparison of methods for solving linear differential equations of fractional order, Chaos, Solitons \& Fractals 31, 1248-1255, 2007.

18. A. Ghorbani, Toward a new analytical method for solving nonlinear fractional differential equations,Comput. Methods Appl. Mech. Eng. 197, 4173-4179, 2008.

19. C. Franke and R. Schaback, Solving partial differential equations by collocation using radial basis functions, Appl. Math. Comput. 93, 73-82, 1998.

20. E.J. Kansa, Multiquadric a scattered data approximation scheme with applications to computational fluid dynamics II. Comput. Math. Appl. 19, 147-61, 1990.

21. T. Tran-Cong, N. Mai-Duy and N. Phan-Thien, BEM-RBF approach for viscoelastic flow analysis, Engineering Analysis with Boundary Elements 26, 757-762, 2002.

22. A.J.M. Ferreira and G.E. Fasshauer, Analysis of natural frequencies of composite plates by an RBF-pseudospectral method, Composite Structures 79, 202-210, 2007.

23. M. Dehghan and A. Shokri, Numerical solution of the nonlinear Klein-Gordon equation using radial basis functions, J. of Computational and Applied Mathematics 230, 400-410, 2009.

24. Q. Shen, A meshless method of lines for the numerical solution of KdV equation using radial basis functions, Engineering Analysis with Boundary Elements 33, 11711180, 2009.

25. S. Karimi Vanani and A. Aminataei, Multiquadric approximation scheme on the numerical solution of delay differential systems of neutral type, Mathematical and Computer Modelling 49, 234-241, 2009.

26. X. Liu, G. R. Liu, K. Tai and K.Y. Lam, Radial point interpolation collocation method for partial differential equations, Computers and Mathematics with Applications 50, 1425-1442, 2005.

27. N. Mai-Duy and T. Tran-Cong, Approximation of function and its derivatives using radial basis function networks, Applied Mathematical Modelling 27, 197-220, 2003.

28. A. Aminataei and M.M. Mazarei, Numerical solution of Poisson's equation using radial basis function networks on the polar coordinate, Computers and Mathematics with Applications 56, 2887-2895, 2008.

29. R.L. Hardy, Multiquadric equations of topography and other irregular surfaces, $J$. Geophys. Res. 76, 1905-1918, 1971.

30. R.L. Hardy, Theory and applications of the multiquadric bi-harmonic method: 20 years of discovery, Comput. Math. Appl. 19, 163-167, (1990).

31. R. Franke, Scattered data interpolation: Tests of some methods, Math. Comput. 38 181-192, 1971.

32. A.H.D. Cheng, M.A. Golberg, E.J. Kansa and G. Zammito, Exponential convergence and h-c multiquadric collocation method for partial differential equations, Numer. Methods Partial Differential Equations 19, 571-594, 2003.

33. C.A. Micchelli, Interpolation of scattered data: distance matrices and conditionally positive definite functions, Constr. Approx. 2, 11-22, 1986.

34. S. Momani and Z. Odibat, Analytical approach to linear fractional partial differential equations arising in fluid mechanics, Physics Letters A 355, 271-279, 2006.

35. Z. Odibat and S. Momani, A reliable treatment of homotopy perturbation method for Klein-Gordon equations, Phys. Lett. A 365, 351-357, 2007. 